\title{
Testing Assumptions about Solute Concentration Dependence in Liquid Crystal NMR
}

\author{
Amandeep S. Taggar, Christopher J. Campbell, Anand Yethiraj,* and E. Elliott Burnell \\ 2036 Main Mall, Chemistry Department, University of British Columbia, Vancouver, \\ British Columbia, V6T 1Z1, Canada
}

Received: May 17, 2005; In Final Form: November 17, 2005

\begin{abstract}
The NMR spectra of four solutes, used as probes of liquid crystal orientational order, were analyzed. For each solute, samples were prepared at different solute concentrations, and the concentration dependence was used to extrapolate zero-concentration properties. The mean-field (Maier-Saupe) model when applied to solutes neglects solute-solute interactions and assumes all solutes in a mixed-solute sample see the same average environment. The first assumption is only valid as one approaches zero concentration, while experiments are typically carried out at concentrations between 0 and $10 \mathrm{~mol} \%$. The solute concentration dependence has in the past been "scaled out" using an internal solute reference as an orientational standard. We measured the concentration dependence of the orientational order parameter and calculate the corresponding interaction energies based on a mean-field interaction potential for a solute. We find agreement at the $3 \%$ level between experiments for different solutes while using (i) the zero-concentration values as solute-dependent orientational references and (ii) scaling to either order parameters or interaction energies; these two scalings gave equivalent but not identical results. We find, too, that errors inherent in the experiment and the calculations will limit attempts to refine the theory to push the comparisons beyond the $2 \%$ level.
\end{abstract}

\section{Introduction}

The presence of orientational order in liquid crystals has led to the wide-spread use of liquid-crystal displays and related applications. It has also attracted interest recently with questions pertaining to biaxiality ${ }^{1,2}$ and the coupling of orientational order to mechanical strain ${ }^{3}$ to create novel elastomeric materials. ${ }^{4,5}$

An important fundamental question concerns the nature of the anisotropic intermolecular forces that contribute to the orientational order. ${ }^{6}$ An excellent way to probe orientational order in liquid crystals is through the use of small solute molecules which themselves become orientationally ordered through their interaction with the anisotropic intermolecular potential of the liquid crystal "solvent". The solute orientational order is readily obtained from its NMR spectrum in a liquid crystal solvent. ${ }^{6-12}$

However, there are problems associated with the use of solutes as probes of liquid crystal properties. The finite concentration of solute usually decreases the liquid crystal/ isotropic transition temperature, leading to decreased liquid crystal orientational order. Models and theories are simplified if they do not have to account for the effect of the solute on the liquid crystal, and are, therefore, normally written in terms of a single solute molecule interacting with the bulk liquid crystal solvent. Ideally, experiments should be performed as a function of concentration in order to extrapolate properly to zero concentration. Such extrapolation is not normal in NMR experiments.

One approach is to use solutes whose symmetry leads to two independent second-rank order parameters, and to compare ratios of these order parameters with theory. ${ }^{13}$ Unfortunately, these ratios are both concentration and temperature dependent, as can be demonstrated using the order parameters reported in ref 14 .

* Present address: Department of Physics and Physical Oceanography, Memorial University of Newfoundland, St. John's, NL, A1B 3X7, Canada.
Thus, results for these solutes at finite concentrations also need to be extrapolated to zero concentration for meaningful comparisons to be made. The investigation of concentration dependence is the subject of this paper and is readily carried out using more symmetrical solutes whose orientational order is described by a single order parameter.

Another approach is to measure the spectra of a collection of solutes and to compare order parameters calculated from the NMR spectra with ones predicted using a model or theory. In such experiments, an attempt is made to ensure that all experiments are performed with solutes at precisely identical conditions, but not at or near the zero-concentration limit. The assumption made is that relative solute orientational order at finite concentration is identical to that at zero concentration.

One successful approach to ensure identical conditions is to codissolve all solutes in the same NMR tube. There are obvious limits on the number and nature of solutes that can be so used because of the complexity of the resulting NMR spectra. ${ }^{15}$ Hence, it is often the case that solute order parameters obtained from different NMR sample tubes must be compared, which raises the question of how to make this comparison. Several approaches have been used. For example, all samples could be run at the same reduced liquid crystal temperature, or all samples could be run at different temperatures for which some chosen solute order parameter (or deuterated liquid crystal deuteron quadrupolar splitting) is kept constant. Alternatively, samples could be run at the same actual temperature, and some chosen solute order parameter (or liquid-crystal quadrupolar splitting) could be used to scale linearly order parameters among samples, or the scaling could be applied to the interaction energies of an assumed mean-field ${ }^{16}$ interaction potential. These ideas were tested with experiments on a collection of aromatic solutes codissolved in the nematic liquid crystal N-(4-ethoxybenzylidene)-2,6-dideutero-4-n-butylaniline (EBBA) using seven different sample tubes, each with differing concentrations of 
solutes. ${ }^{14}$ In these experiments, it was found that the best comparison among samples was obtained from experiments run at the same actual temperature and using the order parameter of the solute 1,3,5-trichlorobenzene (tcb) as an orientational standard for scaling purposes.

An interesting, and possibly puzzling, result is that direct linear scaling using order parameters gave a better comparison than one which involved scaling the interaction energies. It is worthwhile investigating this result using a different liquid crystal. In addition, the total solute concentration varied from 6.1 to 17.2 mol percent. Because most models and theories apply to zero concentration, it would be valuable to extrapolate measurements to zero concentration to see how the extrapolated results compare with those obtained at finite concentration. These ideas are the topic of this paper which uses the liquid crystal mixture Merck ZLI-1132 (see ref 11 for composition).

\section{Experimental Section}

A. Sample Preparation and Calibrations. Each sample was prepared by dissolving one or more of four solutes (benzene, 1,3,5-trifluorobenzene (tfb), tcb , and 1,3,5-tribromobenzene (tbb)) at one of numerous concentrations in the liquid crystal ZLI-1132 in a $5 \mathrm{~mm}$ o.d. high-resolution NMR sample tube; the sample was then thoroughly mixed and sealed.

Two experimental runs were carried out. Run 1 involved solute concentration dependence measurements of four solutes. At this time, the concentration of each solute was varied by sequential addition of the solute to one sample tube (i.e., there was one sample tube for each solute type). Measurements were also made in one "mixed" sample which had all four solutes codissolved.

Run 2 was an independent set of experiments (done on the same NMR spectrometer). In this set of experiments, samples were prepared for two solutes, benzene and tcb, with each solute concentration prepared as a separate sample tube and then flame sealed. This had the advantage that errors in sample preparation were not additive, and the disadvantage that a lot more liquid crystal had to be utilized. These measurements were done at lower solute concentrations to be certain of being in the regime of linear dependence on solute concentration. Three mixed samples were also prepared, two containing the solutes benzene and tcb, and a third containing all four solutes.

All samples were run at a nominal temperature setting of $300.9 \mathrm{~K}$. The airflow was then adjusted to give a splitting of approximately $4450 \mathrm{~Hz}$ in a temperature-standard sample that was used prior to every experiment. The temperature standard to ensure repeatability was a sealed liquid crystalline sample of 10 wt \% benzene in "magic mixture" (55 wt \% ZLI-1132 and 45 wt $\%$ EBBA). ${ }^{6}$ The peak separation measured was not that of the true outermost peaks, but that of the tall peaks adjacent to the outermost peak on each side.

B. NMR Experiments. Proton NMR spectra were measured at a temperature setting of $300.9 \mathrm{~K}$ using a Bruker AMX-500 NMR spectrometer. Samples were allowed an equilibration time of $20 \mathrm{~min}$ inside the probe prior to collection of the spectra. These spectra were then analyzed using the program LEQUOR ${ }^{17}$ to obtain the dipolar couplings of the dissolved solutes benzene, $\mathrm{tfb}$, tcb, and tbb as a function of solute concentration. These raw dipolar couplings are listed in an auxiliary file (see Supporting Information). The program SHAPE ${ }^{18}$ was used to obtain the order matrix for the four solutes from the dipolar couplings. Molecular parameters used for benzene, $\mathrm{tfb}$, and tcb are obtained from the literature. ${ }^{19-21}$ The tbb molecular param-
TABLE 1: Experimental Run 1.

\begin{tabular}{cccc}
\hline solute & mole $\%$ & $\mathrm{~S}_{\mathrm{zz}}(\mathrm{A})^{a}$ & $H_{\mathrm{sol}} / k_{\mathrm{B}} T(\mathrm{~B})^{b}$ \\
\hline benzene & 1.31 & $-0.25942(2)$ & $-1.8274(3)$ \\
benzene & 3.00 & $-0.25225(2)$ & $-1.7428(2)$ \\
benzene & 4.48 & $-0.24787(2)$ & $-1.6963(2)$ \\
benzene & 5.14 & $-0.24414(2)$ & $-1.6575(2)$ \\
benzene & 6.71 & $-0.24184(2)$ & $-1.6340(2)$ \\
benzene & 8.32 & $-0.23599(2)$ & $-1.5755(2)$ \\
benzene & mix 1 & $-0.20010(2)$ & $-1.2507(2)$ \\
tfb & 1.05 & $-0.28295(6)$ & $-2.1054(8)$ \\
tfb & 3.01 & $-0.27645(5)$ & $-2.0226(6)$ \\
tfb & 5.04 & $-0.27013(5)$ & $-1.9454(6)$ \\
tfb & 6.56 & $-0.26483(5)$ & $-1.8831(6)$ \\
tfb & mix1 & $-0.23022(5)$ & $-1.5176(5)$ \\
tcb & 0.85 & $-0.30580(5)$ & $-2.4299(7)$ \\
tcb & 3.29 & $-0.29393(2)$ & $-2.2541(3)$ \\
tcb & 5.54 & $-0.28185(2)$ & $-2.0911(3)$ \\
tcb & 7.19 & $-0.27222(2)$ & $-1.9706(2)$ \\
tcb & 8.94 & $-0.26190(2)$ & $-1.8494(2)$ \\
tcb & 16.45 & $-0.21258(2)$ & $-1.3579(2)$ \\
tcb & mix1 & $-0.24876(2)$ & $-1.7056(2)$ \\
tbb & 1.02 & $-0.30797(2)$ & $-2.4631(3)$ \\
tbb & 2.21 & $-0.30200(2)$ & $-2.3714(3)$ \\
tbb & 3.18 & $-0.29661(2)$ & $-2.2923(3)$ \\
tbb & 4.21 & $-0.29140(2)$ & $-2.2188(3)$ \\
tbb & mix1 & $-0.25096(2)$ & $-1.7290(2)$ \\
t 19 & & &
\end{tabular}

${ }^{a}$ Column A shows order parameters for single-solute samples and one mixed-solute sample. The number in parenthesis indicates the error in the last digit. ${ }^{b}$ Column $\mathrm{B}$ shows the corresponding calculated interaction energies. The values for single-solute samples are also plotted in Figures 1 and 4 for the four solutes benzene, tfb, tcb, and tbb. The mixed-solute sample has the following composition: $3.65 \mathrm{~mol}$ $\%$ benzene, $2.70 \mathrm{~mol} \% \mathrm{tfb}, 4.29 \mathrm{~mol} \% \mathrm{tcb}$, and $2.77 \mathrm{~mol} \% \mathrm{tbb}$.

eters are taken to be identical to those of tcb. We define axes systems in the substituted benzenes as follows: the direction perpendicular to the aromatic ring is the $z$ direction, and the direction along one of the $\mathrm{C}$ halogen bonds is the $x$ direction.

Given the solute molecular parameters, the spectra thus directly yield order parameters, which are our primary measured quantities. Due to the symmetry (symmetry group) of the molecules, we have only one independent order parameter: $S_{z z}$ $=-1 / 2 S_{y y}=-1 / 2 S_{x x}$.

\section{Results}

The results from all experiments in both experimental runs are tabulated in Tables 1 and 2. Measured order parameters are listed in column A in both tables while column B contains the corresponding interaction energies calculated using a mean-field (Maier-Saupe) ${ }^{16}$ interaction potential.

A. Measured Order Parameters. We first plot (Figures 1 and 2) measured order parameter $S_{z z}$ from samples containing a single solute against solute concentration in the liquid crystal ZLI-1132 for each of the four solutes. The order parameter $S_{z z}$ is seen to depend roughly linearly on the solute concentration. The physical quantity of interest is the extrapolation of $S_{z z}$ to zero concentration, where the mean-field picture of a single solute in a pure liquid-crystalline environment with no solutesolute interactions is exact. Previous work ${ }^{14}$ has discussed different methods of "compensating" for the solute's effect at finite concentrations on the liquid crystal phase; in particular, the relative merits of comparing scaled or unscaled order parameters or interaction energies. We will show here that using either order parameters or interaction energies yields comparable results.

Order parameters at "zero-concentration" (column (A) in Table 3) are obtained by linear extrapolation of the concentration dependence of the order parameter of each solute to zero 
TABLE 2: Experimental Run 2

\begin{tabular}{clcl}
\hline solute & mole $\%$ & $\mathrm{~S}_{\mathrm{zz}}(\mathrm{A})^{a}$ & $H_{\mathrm{sol}} / k_{\mathrm{B}} T(\mathrm{~B})^{b}$ \\
\hline benzene & 0.55 & $-0.26276(2)$ & $-1.8592(1)$ \\
benzene & 1.101 & $-0.26135(2)$ & $-1.8432(1)$ \\
benzene & 2.321 & $-0.25619(2)$ & $-1.7856(1)$ \\
benzene & 2.921 & $-0.25390(2)$ & $-1.7606(1)$ \\
benzene & 4.969 & $-0.24643(2)$ & $-1.6812(1)$ \\
benzene & $\operatorname{mix} 2.1$ & $-0.24691(2)$ & $-1.6862(1)$ \\
benzene & $\operatorname{mix} 2.2$ & $-0.24623(2)$ & $-1.6791(1)$ \\
benzene & $\operatorname{mix} 2.3$ & $-0.24877(2)$ & $-1.7057(1)$ \\
tfb & $\operatorname{mix} 2.2$ & $-0.27184(5)$ & $-1.9660(6)$ \\
tcb & 0.599 & $-0.30796(1)$ & $-2.4629(1)$ \\
tcb & 1.087 & $-0.30568(1)$ & $-2.4274(1)$ \\
tcb & 2.375 & $-0.30062(1)$ & $-2.3508(1)$ \\
tcb & 3.099 & $-0.29604(1)$ & $-2.2841(1)$ \\
tcb & 4.755 & $-0.28754(1)$ & $-2.1661(1)$ \\
tcb & $\operatorname{mix} 2.1$ & $-0.29494(1)$ & $-2.2684(1)$ \\
tcb & $\operatorname{mix} 2.2$ & $-0.29464(1)$ & $-2.2641(1)$ \\
tcb & $\operatorname{mix} 2.3$ & $-0.29698(1)$ & $-2.2976(1)$ \\
tbb & $\operatorname{mix} 2.2$ & $-0.29843(1)$ & $-2.3186(1)$
\end{tabular}

${ }^{a}$ Column A shows order parameters for single-solute samples and three mixed-solute samples. ${ }^{b}$ Column B shows the corresponding calculated interaction energies. The values for single-solute samples are also plotted in Figures 2 and 5. Mixed-solute samples have the following compositions (with numbers corresponding to mole \% of benzene/tfb/tcb/tbb): (i) "mix2.1", 2.129/0.000/1.984/0.000; (ii) "mix2.2", 1.748/1.669/0.885/0.510; (iii) "mix2.3", 2.846/0.000/1.060/0.000.

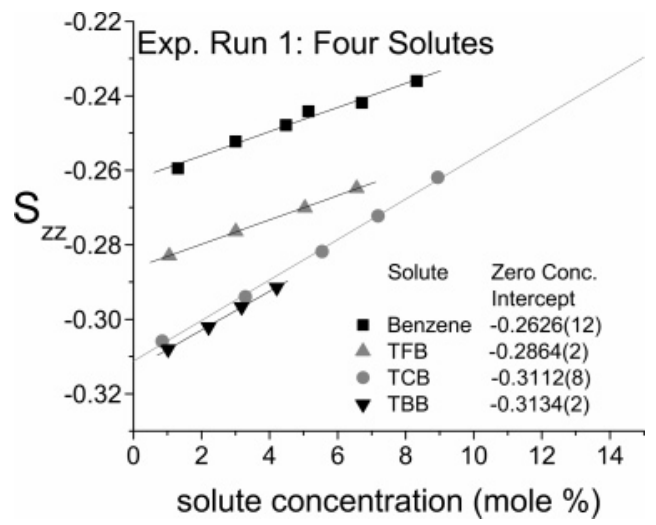

Figure 1. Run 1 experimental order parameters from samples containing a single solute against solute concentration in the liquid crystal ZLI-1132. The zero-concentration intercept is given for each linear fit (the highest concentration point has been omitted for tcb).

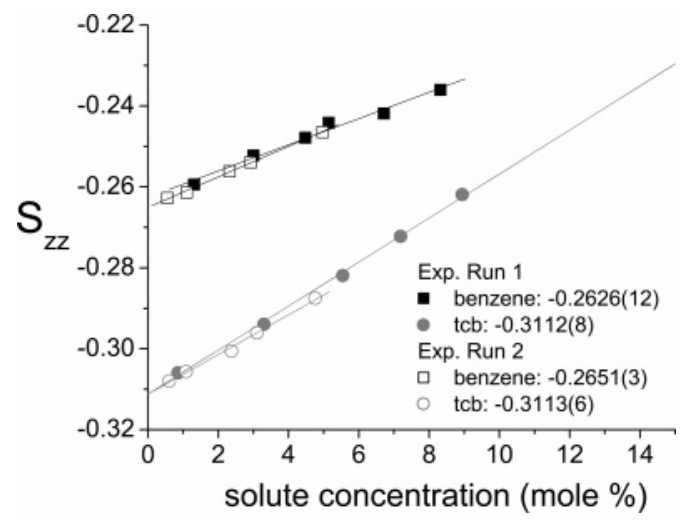

Figure 2. Run 2 experimental order parameters from samples containing a single solute against solute concentration in the liquid crystal ZLI-1132. The benzene and tcb results from Run 1 are repeated for comparison. The zero-concentration intercept is given for each linear fit.

concentration (Figures 1 and 2). For each solute in each experimental run, the ratio of the order parameter of a solute in a mixed-solute sample (column B in Table 3) to its value at
TABLE 3

\begin{tabular}{|c|c|c|c|}
\hline & $\begin{array}{l}S_{\mathrm{zz}, \mathrm{zc}} \\
(\mathrm{A})^{a}\end{array}$ & $\begin{array}{l}\mathrm{S}_{\mathrm{zz}, \operatorname{mix}} \\
(\mathrm{B})^{b}\end{array}$ & $\begin{aligned} R_{S}= & S_{z z, z c} / S_{z z, m i x} \\
(C)^{\mathrm{c}} & \end{aligned}$ \\
\hline $\begin{array}{l}\text { benzene } \\
\text { tfb } \\
\text { tcb } \\
\text { tbb }\end{array}$ & $\begin{array}{l}-0.2626(12) \\
-0.2864(2) \\
-0.3112(8) \\
-0.3134(4)\end{array}$ & $\begin{array}{l}\text { Exp. Run } 1 \\
\operatorname{mix} 1:-0.20010(2) \\
\operatorname{mix} 1:-0.23002(5) \\
\operatorname{mix} 1:-0.24876(2) \\
\operatorname{mix} 1:-0.25096(2)\end{array}$ & $\begin{array}{l}1.312(8) \\
1.245(6) \\
1.251(2) \\
1.249(7)\end{array}$ \\
\hline benzene & $-0.2651(3)$ & $\begin{array}{l}\text { Exp. Run } 2 \\
\operatorname{mix} 2.1:-0.24691(2) \\
\operatorname{mix} 2.2:-0.24623(2) \\
\operatorname{mix} 2.3:-0.24877(2) \\
\operatorname{mix} 2.2:-0.27184(1) \\
\operatorname{mix} 2.1:-0.29494(1) \\
\operatorname{mix} 2.2:-0.29464(1) \\
\operatorname{mix} 2.3:-0.29698(1) \\
\operatorname{mix} 2.2:-0.29843(1)\end{array}$ & $\begin{array}{l}1.074(2) \\
1.077(2) \\
1.066(2) \\
1.054(1 *) \\
1.055(2) \\
1.057(2) \\
1.048(2) \\
1.050(1 *)\end{array}$ \\
\hline
\end{tabular}

${ }^{a}$ Column A: zero concentration order parameters $S_{z z, z c}$ (extrapolations from Figures 1 and 2) for the four solutes benzene, tfb, tcb, and tbb. ${ }^{b}$ Column B: order parameters $S_{z z \text {,mix }}$ in different two- and four-solute mixtures. ${ }^{c}$ Column C: the ratio $R_{S}$ of values in column A to column $\mathrm{B}$; the asterisk indicates solutes $\mathrm{tfb}$ and tbb, where zero-concentration values from experimental run 1 were used to calculate the ratios for Run 2.

zero-concentration (column A in Table 3 ) is tabulated (column $\mathrm{C}$ in Table 3).

B. Calculated Interaction Energies. We assume that anisotropic interactions between solute and liquid crystal are described by the simple mean-field ${ }^{16}$ interaction potential. Interaction energies may then be calculated from the order parameters. For a homogeneous uniaxial nematic, the mean-field potential is given as

$$
H_{l c}=-v_{l c}\left\{S_{l c} P_{2}(\cos \theta)\right\}
$$

where $v_{l c}$ is a scale parameter and $S_{l c}=\left\langle P_{2}(\cos \theta)\right\rangle$ is the nematic order parameter. Experimental observations of solutes in a given nematic liquid crystal can be modeled by a mean-field potential involving a tensorial solute molecular property $\beta_{\gamma \delta}^{\text {total }}$ whose anisotropic, traceless part $\beta_{\gamma \delta}$ interacts with an average liquid crystal field $F_{\gamma \delta}$ :

$$
H_{\mathrm{sol}}=-1 / 2 F_{\gamma \delta} \beta_{\gamma \delta}
$$

This interaction is the lowest-order nonzero term in an expansion of any short-range interaction for nematic liquid crystals. $\mathrm{F}_{\gamma \delta}$ and $\beta_{\gamma \delta}^{\text {total }}$ have been identified physically as interactions such as that between a mean electric field (squared) and a dielectric polarizability, or between a liquid crystal electric field gradient and a solute quadrupole moment tensor. ${ }^{6}$ For the purposes of this paper, the precise nature of the anisotropic intermolecular interactions is not important. For a solute, whose symmetry leads to two independent second-rank order parameters (a "biaxial" solute) in a uniaxial nematic phase, eq 2 becomes

$$
H_{\text {sol }}=-3 / 4 F_{z z} \beta_{z z} P_{2, \text { asymm }}
$$

where $P_{2 \text {,asymm }}=P_{2}\left(\cos \theta^{\prime}\right)-(b / 2) \sin ^{2} \theta^{\prime} \cos (2 \phi)$. Here we have defined the asymmetry in the solute molecular tensor

$$
b=\left(\beta_{x x}-\beta_{y y}\right) / \beta_{z z}
$$

and $x, y$, and $z$ are chosen along the principal axis of the $\beta$ tensor. The magnetic field is along $Z$ and $x, y, z$ and $X, Y, Z$ are molecule-fixed and lab-fixed coordinates $\left(z . Z \equiv \cos \left(\theta^{\prime}\right)\right.$ and $\boldsymbol{x} . \boldsymbol{Z}$ $\left.\equiv \sin \left(\theta^{\prime}\right) \cos (\phi)\right)$. For a solute whose symmetry leads to only one independent second-rank order parameter, $b=0$, and 


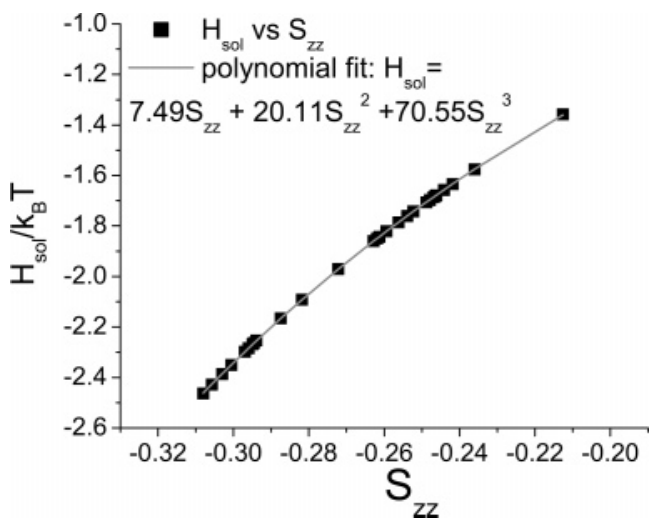

Figure 3. Interaction energy vs order parameter. The solid symbols are values for the solutes benzene and tcb. The line is the fit to a secondorder polynomial.

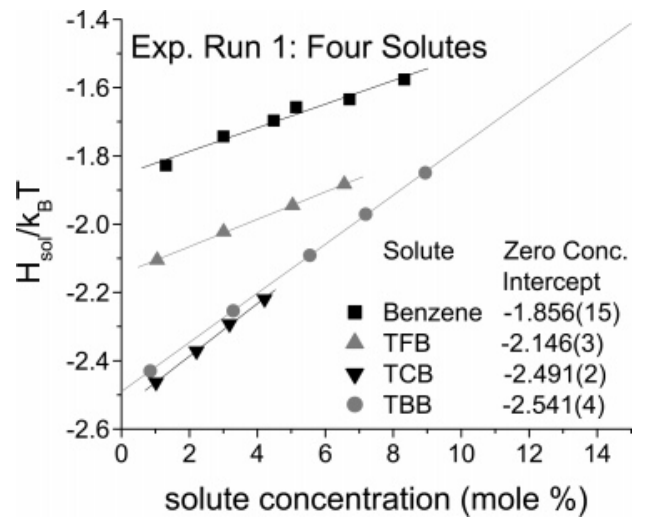

Figure 4. Run 1 interaction energies from samples containing a single solute against solute concentration in the liquid crystal ZLI-1132. The zero-concentration intercept is given for each linear fit (the highest concentration point has been omitted for tcb).

eq 3 reduces to

$$
H_{\mathrm{sol}}=-3 / 4 F_{\mathrm{zZ}} \beta_{z z} P_{2}\left(\cos \theta^{\prime}\right)
$$

The order parameter is calculated from the above model potential (eq 5) using the relation:

$$
S_{z z}=Z^{-1} \int P_{2}\left(\cos \theta^{\prime}\right) \exp \left[-H_{\mathrm{sol}} / k_{B} T\right] \sin \theta^{\prime} d \theta^{\prime}
$$

where $Z=\int \exp \left[-H_{\mathrm{sol}} / k_{B} T\right] \sin \theta^{\prime} d \theta^{\prime}$ is the partition function. There is thus a simple (but not linear) functional relationship between observed order parameter and the calculated interaction energy that fits it. This relationship is shown in Figure 3, where each solid symbol represents $\left(S_{z z}, H_{s o l}\right)$ values for solutes benzene or tcb (the $H_{\text {sol }}$ being calculated using eq 6 by fitting to the experimental solute order parameter $S_{z z}$ ); the line is a fit to a polynomial with linear, quadratic, and cubic terms. Note that the numerical value of the sum of the nonlinear terms is roughly of the same magnitude as that of the linear term; thus the scaling of $S_{z z}$ and of $H_{\text {sol }}$ are inequivalent. The sole purpose of the polynomial fit is to show the nonlinear relationship between $S_{z z}$ and $H_{\text {sol }}$. We ascribe no physical significance to this fit.

The run 1 solute concentration dependence of calculated interaction energies of solutes benzene, $\mathrm{tfb}$, tcb, and tbb, are shown in Figure 4. Comparisons of results for the two runs for the solutes tcb and benzene are shown in Figure 5.

The calculated interaction energies at "zero-concentration" (column A in Table 4) are obtained by linear extrapolation of the concentration dependence of the interaction energies of each

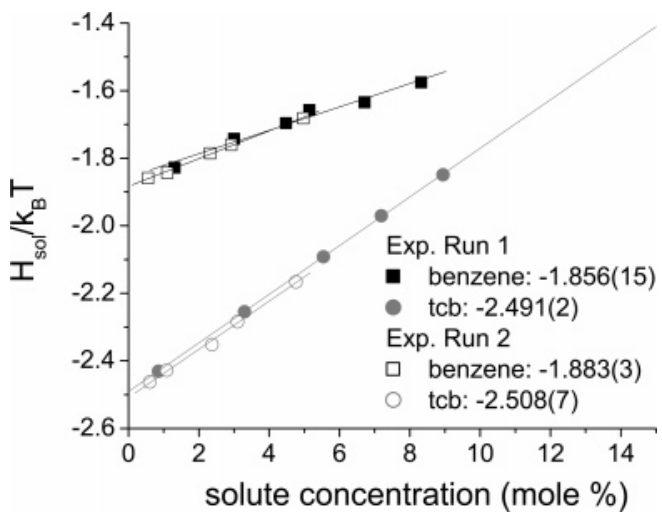

Figure 5. Run 2 interaction energies from samples containing a single solute against solute concentration in the liquid crystal ZLI-1132. The benzene and tcb results from run 1 are repeated for comparison. The zero-concentration intercept is given for each linear fit.

\begin{tabular}{|c|c|c|c|}
\hline & $\begin{array}{c}H_{s o l, z c} / k_{B} T \\
(\mathrm{~A})^{a}\end{array}$ & $\begin{array}{l}H_{\text {sol,mix }} / k_{B} T \\
\text { (B) })^{b}\end{array}$ & $\begin{array}{c}R_{H}=H_{\text {sol, }, z} d H_{\text {sol,mix }} \\
(\mathrm{C})^{c}\end{array}$ \\
\hline $\begin{array}{l}\text { benzene } \\
\text { tfb } \\
\text { tcb } \\
\text { tbb }\end{array}$ & $\begin{array}{l}-1.856(15) \\
-2.146(3) \\
-2.491(2) \\
-2.541(4)\end{array}$ & $\begin{array}{c}\text { Exp. Run } 1 \\
\operatorname{mix} 1:-1.2507(2) \\
\operatorname{mix} 1:-1.5176(5) \\
\operatorname{mix} 1:-1.7056(2) \\
\operatorname{mix} 1:-1.7290(2)\end{array}$ & $\begin{array}{l}1.484(7) \\
1.414(2) \\
1.460(2) \\
1.470(3)\end{array}$ \\
\hline benzene & $-2.508(6)$ & $\begin{array}{l}\text { Exp. Run } 2 \\
\operatorname{mix} 2.1:-1.6862(1) \\
\operatorname{mix} 2.2:-1.6791(1) \\
\operatorname{mix} 2.3:-1.7057(1) \\
\operatorname{mix} 2.2:-1.9660 \\
\operatorname{mix} 2.1:-2.2684(1) \\
\operatorname{mix} 2.2:-2.2641(1) \\
\operatorname{mix} 2.3:-2.2976(1) \\
\operatorname{mix} 2.2:-2.3186\end{array}$ & $\begin{array}{l}1.117(3) \\
1.121(3) \\
1.104(3) \\
1.092(*) \\
1.106(3) \\
1.108(3) \\
1.092(3) \\
1.096(*)\end{array}$ \\
\hline
\end{tabular}

TABLE 4

${ }^{a}$ Column A shows zero concentration interaction energies $H_{\text {sol, } z c}$ $k_{B} T$ (extrapolations from Figures 4 and 5) for the four solutes benzene, tfb, tcb and tbb. ${ }^{b}$ Column B shows interaction energies $H_{s o l, m i x} / k_{B} T$ in different two- and four-solute mixtures. ${ }^{c}$ Column $\mathrm{C}$ shows the ratio $R_{H}$ of values in column A to column B; the asterisk indicates solutes $\mathrm{tfb}$ and tbb, where zero-concentration values from experimental run 1 were used to calculate the ratios for run 2 .

solute to zero concentration (Figures 4 and 5). Note that while the concentration dependence of the order parameter and the energy are both roughly linear, linearity of one does not imply linearity of the other. Therefore, obtaining the zero-concentration interaction energy by linear extrapolation is not equivalent to converting the zero-concentration order parameter to interaction energy.

For each solute in each experimental run, the ratio of the interaction energy of a solute in a mixed-solute sample to its value at zero-concentration is tabulated (column $\mathrm{C}$ in Table 4).

\section{Contributions to Errors}

It is appropriate to summarize briefly the different contributions to errors that we have encountered.

First, sample preparation gave rise to a possible systematic and statistical error in solute concentration, arising from errors in weighing. The statistical errors involved in weighing a few milligrams of solute were $0.5 \mathrm{mg}$ in experimental run 1 and improved in experimental run 2 to $0.1 \mathrm{mg}$ (this improvement enabled confirmation of concentration dependence at low concentrations). An important concern in sample preparation was the volatility of the liquids benzene and $t f b$. In experimental run 1 , higher-solute concentration samples were prepared by 
sequential addition of solute to the same NMR tube. This, coupled with the volatility of benzene and $\mathrm{tfb}$, gave rise to the possibility of a systematic underestimation of solute concentration. Hence, in experimental run 2, a sample was made in parallel for each solute-concentration in a separate NMR tube, and immediately sealed. In retrospect, the consistency between the two runs suggests that solute volatility did not affect our results.

Second, the spectra were collected at "constant temperature". The temperature standard to ensure repeatability was a liquid crystalline sample of $10 \mathrm{wt} \%$ benzene in "magic mixture" (55 wt \% ZLI-1132 and 45 wt \% EBBA). The temperature setting and/or the air flow was adjusted to give a peak splitting of 4450 $\mathrm{Hz}$. The estimated error was $2 \mathrm{~Hz}$, which corresponded to a temperature variation of $\approx 0.05 \mathrm{~K}$.

Third, the dipolar couplings for tcb and tbb (both spectra are a 1:2:1 triplet) are one-sixth the splitting between the two outer peaks, and are unambiguously determined. For the many-peak $\mathrm{tfb}$ and benzene spectra, the conversion from spectrum to the dipolar coupling matrix depends on the choice of $\mathbf{J}$ couplings used. In addition, possible anisotropies in J couplings involving F nuclei ${ }^{22}$ in $\mathrm{tfb}$ are neglected. In this case, the value of $D_{H H}$ is directly proportional to the order parameter, and for all tfb spectra, $D_{H H}$ is fitted to better than $0.05 \mathrm{~Hz}$ in the SHAPE program. Hence any anisotropy in $J_{F F}$ (or any incorrect value of $J_{H F}$ ) has negligible effect on the order parameters obtained. Note that an indication of possible anisotropy in the $J_{F F}$ indirect coupling is that in all cases $D_{F F}$ (experimental) minus $D_{F F}$ (calculated) is of the order $3 \mathrm{~Hz}$; however, this difference could also arise from neglect of reorientation-vibration effects. ${ }^{23}$

Fourth, molecular structure parameters are inputs for the calculation of order parameters from the dipolar couplings (the SHAPE program). Again, different, roughly equivalent, choices of bond lengths can give rise to order parameter differences of approximately $1 \%$. The choice of molecular structural parameters is taken from reported literature values: benzene, ${ }^{19} \mathrm{tfb},{ }^{20}$ tcb. ${ }^{21}$ Indeed all structures are obtained for the solute molecules in an isotropic environment, and it is reasonable to expect that in the absence of corrections for both molecular vibrations and reorientation-vibration effects, ${ }^{23}$ the structure in an anisotropic environment might appear to be slightly different. Again, however, consistent use of the same molecular parameters for all samples ensures that relative comparisons between them are unaffected by this choice. For example, the ratios of dipolar couplings in $\mathrm{tfb}\left(D^{13} / D^{12}=0.2335, D^{14} / D^{12}=0.1263\right.$, and $D^{24} /$ $\mathrm{D}^{12}=0.1582$ ) show a standard deviation of $0.03 \%$ across different samples at different concentrations of $\mathrm{tfb}$. Hence it is important to take note of molecular parameters when making quantitative comparisons, to better than $1 \%$, of order parameters (even those scaled to a solute reference such as tcb) to the results of other experiments. In this work, where the same solute was used, the molecular parameters used are identical to those in ref 14 .

Finally, errors in extrapolations of solute-concentration dependence (of order parameters or energies) have a larger associated statistical error $(\approx 0.2 \%)$ than individual measurements $(\approx 0.01 \%$ or better $)$. It is noted that the errors in the linear extrapolation of energies to zero concentration are larger (by $40 \%-80 \%$ ) than the linear extrapolation of order parameters to zero concentration. An additional problem is associated with the assumption that the extrapolations in Figures 1, 2, 4, and 5 are linear; the results do not justify fitting to a different form, but an unknown error should be associated with the linear
TABLE 5: Order Parameters and Energies that Are Scaled to the Corresponding tcb Value, for Both Extrapolated Zero-concentration Values as Well as for Finite-concentration Mixed-solute Samples ${ }^{a}$

\begin{tabular}{|c|c|c|c|c|c|c|}
\hline & \multicolumn{2}{|c|}{ Exp. Run 1} & \multicolumn{4}{|c|}{ Exp. Run 2} \\
\hline & zero-conc & $\operatorname{mix} 1$ & zero-conc & $\operatorname{mix} 2.1$ & $\operatorname{mix} 2.2$ & $\operatorname{mix} 2$ \\
\hline & 0.8438 & 0.8044 & 0.8516 & 0.8372 & 0.8357 & .83 \\
\hline & 0.9203 & 0.9247 & & & 0.9226 & \\
\hline$t, t b b / S_{z z, c b}$ & 1.0071 & 1.0089 & & & 1.0129 & \\
\hline$H_{b e n z} / H_{t c b}$ & 0.7451 & 0.7331 & 0.7508 & 0.7434 & 0.7416 & 0.74 \\
\hline$H_{t f b} / H_{t c b}$ & 0.8615 & 0.8896 & & & 0.8683 & \\
\hline$H_{t b b} / H_{t c b}$ & 1.0201 & 1.0134 & & & 1.0241 & \\
\hline
\end{tabular}

${ }^{a}$ Scaling of order parameters to the tcb order parameter has been proposed as a way to remove, at least partially, the effects of working with finite-concentration samples.

assumption. Note that the highest concentration tcb point is omitted from the linear fits.

\section{Discussion}

The assumption that has often been used is that, in mixtures, different solutes see the same average anisotropic environment, and thus comparisons with theories and models can be made in terms of ratios of some measure of solute orientational order (such as order parameter $S_{z z}$ or anisotropic interaction energy $H$ ). Since the models and theories normally apply to infinite dilution, a useful test of this assumption is comparison of ratios $R_{S}$ of solute order parameter (or $R_{H}$ of solute interaction energy) in a mixed solute sample to that at zero concentration. Such ratios are reported in Tables 3 (for order parameters) and 4 (for interaction energies) for the four solutes benzene, tfb, tcb, and tbb in ZLI-1132. The test is that $R_{S}$ (or $R_{H}$ ) values for different solutes in the same mixture should be equal. The $R_{S}$ and $R_{H}$ ratios for experimental run 1 agree to $3 \%$ and $2 \%$, respectively; all ratios agree to $1 \%$ for the three mixtures used in experimental run 2 where the mixtures were at lower overall solute concentration. The run $1 R_{S}$ values for benzene are $5 \%$ larger than the average for all solutes, while the $R_{H}$ values are only $1.6 \%$ larger. The $R_{S}$ value for $\mathrm{tfb}$ is roughly equal the values for tcb and tbb, while the $R_{H}$ value is lower. The run 2 , mix 2.2 values for benzene are $1.9 \%$ (for $R_{S}$ ) and $1.2 \%$ (for $R_{H}$ ) larger than the average of the other solutes. These results indicate that, in the current experiments, lower solute concentration mixtures give results that are closer to the zero-concentration limit than do higher concentration mixtures. In addition, using interaction energies for comparisons appears, in this case, to be preferable to using order parameters.

Since most experiments are done at finite, and sometimes large, solute concentrations, different scalings have been proposed to hopefully "scale" out the concentration dependence. A previous work using the liquid crystal $\mathrm{EBBA}^{14}$ reported that conducting experiments at a constant temperature and then scaling the order parameter (as opposed to the energy) with respect to tcb provided the most consistent results (the results with the lowest standard deviation). We explore this in Table 5 by comparing ratios of both order parameters and energies to the values in tcb. This is done for all the mixed-solute samples (as is typical) and for the zero-concentration extrapolations (new to this work and, in principle, the quantity of relevance to models and theories). The test is that ratios of $S_{\text {solute }} / S_{\text {tcb }}$ (or $H_{\text {solute }} / H_{\text {tcb }}$ ) obtained from different mixtures and from zero-concentration extrapolations (i.e., along any given row of Table 5) should be equal.

In general, the values for mixtures are close to the values at zero-concentration, and the relative spread between mixed- 
sample values and zero-concentration values is more uniformly small $(\approx 1 \%)$ in the energy ratios for both experimental runs. The largest deviation is for the order parameter ratio for benzene in run 1 . The energy comparison and the run 2 mixture results for benzene are more consistent. For $\mathrm{tfb}$, the $\mathrm{S}$ comparison is somewhat better than the $\mathrm{H}$ comparison.

We have studied four aromatic solutes at various concentrations in the liquid crystal ZLI-1132 in order to investigate the most appropriate manner in which experimental order parameters should be compared with theories and models. The ideal would be to extrapolate to zero concentration in each case. However, such a procedure does have problems, including the dangers associated with the extrapolation process and the need to collect and analyze (potentially) complicated spectra at many concentrations. We have shown that using samples where many solutes are codissolved in the same NMR tube gives results that are similar to zero-concentration ones.

In the case of ZLI-1132 it appears that the method of choice for scaling results among different sample tubes is to scale the solute interaction energies using some chosen solute (such as tcb) as an orientational standard. Using the energies makes physical sense in terms of all solutes experiencing an identical mean field. However, previous results argued that the method of choice for EBBA is to use order parameters for this purpose. Both studies utilized a collection of aromatic solutes that were relatively well ordered. It would be interesting to investigate in both ZLI-1132 and EBBA a series of solutes that have more of a spread in order parameters, and especially to include one (or more) that is significantly more ordered than those studied here. The variation in orientational order should facilitate the use of the nonlinear relationship between $S$ and $H$, as shown in Figure 3 , to settle the question whether $S$ or $H$ is the appropriate parameter to use for scaling. It would also be interesting to expand this work to include the concentration and temperature dependence of solutes in additional liquid crystals, and also to investigate the ratio of the order parameters for biaxial solutes.

One thing is clear, comparison at the 5\% level between experiment and theory (or model calculation) of order parameters is readily achieved using an orientational standard (such as tcb), and scaling to either order parameters or interaction energies. However, great care is necessary if one wishes to push the comparisons beyond the $2 \%$ level.
Acknowledgment. We acknowledge the Natural Sciences and Engineering Research Council of Canada for financial support and E. Merck, Darmstadt, Germany for the gift of ZLI1132 .

Supporting Information Available: Tables of experimental dipolar couplings. This material is available free of charge via the Internet at http://pubs.acs.org.

\section{References and Notes}

(1) Madsen, L. A.; Dingemans, T. J.; Nakata, M.; Samulski, E. T. Phys. Rev. Lett. 2004, $92,145505$.

(2) Acharya, B. R.; Primak, A.; Kumar, S. Phys. Rev. Lett. 2004, 92 , 145506

(3) Finkelmann, H.; Kock, H.; Rehage, G. Makromol. Chem. Rapid Сотmun. 1981, 2, 317

(4) Finkelmann, H.; Kim, S. T.; Munoz, A.; Palffy-Muhoray, P.; Taheri, B. Adv. Mater. 2001, 13, 1069 .

(5) Shenoy, D. K.; Thomsen, D. L., III; Srinivasan, A.; Keller, P.; Ratna, B. R. Sens. Actuators A 2002, 96, 184.

(6) NMR of Ordered Liquids; Burnell, E. E.; de Lange, C. A., Eds.; Kluwer Academic: Dordrecht, The Netherlands, 2003.

(7) Saupe, A.; Englert, G. Phys. Rev. Lett. 1963, 11, 462.

(8) Buckingham, A. D.; McLauchlan, K. A. Prog. Nucl. Magn. Reson. Spectrosc. 1967, 2, 63

(9) Diehl, P.; Khetrapal, C. L. NMR, Basic Principles and Progress Springer: Berlin, 1969; Vol. 1

(10) Emsley, J. W.; Lindon, J. C. NMR Spectroscopy using Liquid Crystalline Solvents; Pergamon Press: Elmsford, NY, 1975.

(11) Lounila, J.; Jokisaari, J. Prog. Nucl. Magn. Reson. Spectrosc. 1982, 15,249

(12) Burnell, E. E.; de Lange, C. A. Chem. Rev. 1998, 98, 2359.

(13) Luckhurst, G. R. In Nuclear Magnetic Resonance of Liquid Crystals; Emlsey, J. W., Ed.; NATO Advanced Study Institute Series C, vol. 141, p $62-65$.

(14) Syvitski, R. T.; Pau, M. Y.-M.; Burnell, E. E. J. Chem. Phys. 2002, $117,376$.

(15) Syvitski, R. T.; Burnell, E. E. J. Magn. Reson. 2000, 144, 58.

(16) Maier, W.; Saupe, A. Z. Naturforsch. 1958, A13, 564; 1959, A14, $882 ; 1960, A 15,287$

(17) Diehl, P.; Kellerhals, H.; Lustig, E. NMR, Basic Principles and Progress; Springer-Verlag: 1972; Vol. 6.

(18) Diehl, P.; Heinrichs, P. M.; Niederberger, W. Mol. Phys. 1971, 20,139 .

(19) Diehl, P.; Niederberger, W. J. Magn. Reson. 1973, 9, 495.

(20) Romondo, F.; Portalone, G.; Domenicano, A.; Schultz, G.; Hargittai, I. J. Mol. Struct. 1992, 269, 367.

(21) Almenningen, A.; Hargittai, I.; Brunvoll, J.; Domenicano, A.; Samdal, S. J. Mol. Struct. 1984, 116, 199.

(22) Vaara, J.; Kaski, J.; Jokisaari, J. J. Phys. Chem. A 1999, 103, 5675.

(23) Snijders, J. G.; de Lange, C. A.; Burnell, E. E. Isr. J. Chem. 1983, 23,269 . 